Ann. Sci. forest., 1967, 24 (3), 205-231.

\title{
OBSERVATIONS SUR L'ANISOTROPIE DU PIN MARITIME DES LANDES
}

\author{
H. POLGE et G. ILLY \\ Station de Recherches sur la Qualité des Bois, \\ Centre national de Recherches forestières, 54 - Nancy \\ Station de Recherches forestières, 33 - Bordeaux \\ Institut national de la Recherche agronomique
}

\section{SOMMAIRE}

Les pins maritimes des Landes sont caractérisés par une forte anisotropie qui est en général plus marquée dans le sens Est-Ouest.

Le côté Est a notamment des cernes plus larges et du bois plus dense, et présente la plupart des autres critères distinctifs du bois de compression, à l'exception d'un retrait longitudinal trop élevé. L'anisotropie des densités et celle des largeurs de cernes sont cependant faiblement liées entre elles ; la première parait occasionnée par les vents dominants venant de l'Ouest, tandis que la seconde dépendrait davantage de l'inclinaison des füts.

L'étude de l'héritabilité et des corrélations génétiques permet de déterminer dansquelle mesure l'anisotropie pourrait être réduite à l'aide des techniques habituelles d'amélioration des arbres forestiers, et de prévoir les conséquences, favorables ou défavorables, qui en résulteraient sur les autres caractéristiques de croissance ou de qualité du bois.

Les forestiers landais se sont, depuis longtemps, rendu compte que le bois formé sur la face Est des Pins maritimes différait sensiblement au point de vue aspect extérieur, structure et propriétés, de celui de la face opposée : les résiniers par exemple savent bien que le " brus », c'est-à-dire la partie renflée située en général du côté Est, leur fournit de meilleurs rendements en gemme, mais qu'il est par contre plus dur, puisqu'il était autrefois nécessaire, en gemmage traditionnel, d'y aiguiser plus souvent l'outil qui servait à entailler le bois, ou " hapchot $»$. Pour les scieurs, le côté « brus ", à accroissements nettement plus larges, présente l'intérêt d'offrir une

Noté : Des éléments de cet article ont fait lobjet d'une communication faite au Congrès de l'Association française pour l'avancement des Sciences, Bordeaux, juillet 1967. 
zone plus importante, nette de nœuds, et de permettre ainsi une production plus élevée de bois de choix, en supposant bien entendu que l'élagage, naturel ou artificiel, soit intervenu la même année sur toutes les faces des arbres.

Cependant, hormis ces quelques constatations empiriques, il apparaît que les divers phénomènes, se rattachant aux variations de l'ensemble des caractéristiques du bois suivant l'orientement, ont été assez peu étudiés jusqu'ici.

En première analyse, on peut penser que le bois formé du côté Est des Pins maritimes des Landes s'apparente d'assez près au bois de compression ; nous commencerons donc par rappeler les principales propriétés de ce bois, ainsi que les hypothèses qui ont été émises pour en expliquer la production, avant d'étudier de façon plus approfondie l'anisotropie du Pin maritime dans le massif landais, et de voir jusqu'à quel point cette anisotropie peut s'expliquer par la présence sur le côté Est de bois de compression.

\section{I. - RAPPELS SUR LE BOIS DE COMPRESSION}

\section{1. - CARACTÉRISTIQUES}

Le bois de compression a déjà fait l'objet de nombreuses études, et une abondante littérature existe à son sujet ; une excellente synthèse des principaux phénomènes le concernant peut, en particulier, être trouvée dans DADSWELL et WARDRoP (1949).

Nous rappellerons seulement que le bois de compression est d'ordinaire plus foncé, plus dense et plus dur que le bois normal ; il apparaît généralement chez les résineux à la partie inférieure des branches ou des tiges penchées ; il s'y traduit par des cernes de plus grande largeur, en sorte que leurs sections sont excentrées et que leur moelle se trouve déportée vers la partie supérieure; de plus dans les cernes larges et denses du bois de compression, la transition entre bois de printemps et bois d'été est très progressive, même pour les essences dans lesquelles le passage de l'un à l'autre est extrêmement brutal en son absence.

A l'échelle microscopique, le bois de compression est caractérisé par la présence de trachéides plus ou moins arrondies, souvent séparées les unes des autres par des méats intercellulaires. En section longitudinale, les parois des trachéides apparaissent comme striées, et il a été prouvé que ces stries correspondent à l'orientation des microfibrilles de la couche moyenne de la membrane secondaire qui sont disposées en spirales relativement aplaties alors que, dans le bois normal, elles sont presque parallèles à l'axe des fibres ; les trachéides du bois de compression sont généralement plus courtes, et ont une teneur en lignine plus élevée, particulièrement en ce qui concerne la membrane secondaire.

Au point de vue technologique, la caractéristique essentielle du bois de compression est son retrait longitudinal élevé, pouvant atteindre 3 ou même $4 \%$, alors que celui du bois normal ne dépasse généralement pas $0,1 \%$ entre l'état frais et l'état anhydre ; on admet le plus souvent qu'en contrepartie le retrait tangentiel du bois de compression est inférieur à celui du bois normal de même densité. 
En ce qui concerne les qualités mécaniques enfin, le bois de compression se différencie, toutes choses égales par ailleurs, par une baisse du module d'élasticité, ainsi que de la résistance au choc et à la traction.

\section{2. - MÉCANISME DE FORMATION DU BOIS DE COMPRESSION}

Si l'on courbe la tige d'un jeune résineux au point d'en faire une boucle, du bois de compression se forme à la partie inférieure, aussi bien dans la zone tendue que dans la partie comprimée de la boucle ; cette expérience fondamentale (EwART et MASSONJONES, 1906) montre que le terme « bois de compression $»$ ne convient guère puisque, dans la partie inférieure de la boucle, ce bois anormal correspond à la zone tendue ; on en déduit que le phénomène en cause n'est pas lié à la compression elle-même, mais à la gravité, les deux forces agissant seulement dans le même sens lorsque l'on a affaire à une tige qui, tout en demeurant rectiligne, s'écarte plus ou moins de la verticale.

On a pensé également à une action possible de la lumière en observant que le bois de compression se rencontrait généralement du côté des tiges opposé à l'éclairement naturel ; cette hypothèse ne se vérifie pas (expérience de WerSHING et BAILEY, 1942) lorsqu'on fait pousser la tête en bas un jeune plant de résineux et qu'on l'éclaire par en-dessous : le bois de compression continue à se produire à la partie inférieure des branches, c'est-à-dire du côté où s'exerce l'attraction terrestre, et non pas du côté opposé à la lumière.

Au reste, les forces de gravité ne sont pas les seules qui soient susceptibles de provoquer la formation de bois de compression, puisqu'en soumettant un plant à une force centrifuge radiale, du bois de compression apparaît du côté opposé (JACCARD, 1938).

La question se pose de savoir si ces diverses forces agissent de façon directe ou indirecte ; en l'état actuel des expériences, c'est la deuxième hypothèse qui semble prévaloir, puisque, en exerçant directement une force longitudinale de compression ou de traction sur une tige maintenue verticale, BUSGEN et MUNCH (1929) n'ont observé aucune modification anatomique, que ce soit du côté où la force est appliquée, ou du côté opposé.

Pour cette raison, on pense que la formation de bois de compression n'est qu'une conséquence indirecte des contraintes auxquelles l'arbre est soumis, et qu'elle résulte en fait d'un mécanisme hormonal, la distribution de l'auxine se trouvant modifiée par suite des phénomènes mécaniques évoqués plus haut ; en particulier chez les Gymnospermes, Strasburger (1891) a observé que le développement des bourgeons est plus important à la partie inférieure des tiges penchées ; l'activité cambiale y commençant de surcroît au printemps plus tôt que du côté opposé, une plus grande quantité d'auxine parviendrait à l'assise génératrice, entrainant la production de cellules plus nombreuses à division plus rapide ; cette hypothèse, qui est maintenant admise par la quasi-totalité des auteurs, se trouve confirmée par l'expérience : lorsqu'on procède, comme l'on fait Wershing et BaILeY (op. cit.), à une application d'acide indol- $\beta$-acétique sur l'un des côtés d'une tige de résineux, du bois de compression se forme dans la zone intéressée. 
Parmi les facteurs qui peuvent entraîner la production de bois de compression, on doit mentionner plus particulièrement le vent, lorsqu'il souffle toujours, ou presque toujours, dans le même sens, mais son action n'est pas simple : dans une expérience récente sur Mélèzes, LARSON (1965) a montré que, si l'on soumet de jeunes plants résineux à un vent de direction constante, les accroissements annuels augmentent de largeur à la partie inférieure du côté opposé au vent oủ ils sont alors constitués par du bois de compression ; ceci se produit d'ailleurs aux dépens des niveaux supérieurs, où les cernes sont, au contraire, moins larges que ceux des témoins. Lorsque les plants sont tuteurés, l'exposition au vent reste sans effet notable sur l'épaisseur des couches annuelles. Quant à la croissance en hauteur, elle diminue dans les plants exposés au vent latéral, mais se rapproche de la normale chez ceux qui sont assujétis à un tuteur.

Si la direction du vent est variable, la largeur des accroissements annuels se trouve également augmentée à la partie inférieure, mais dans toutes les directions, et le bois élaboré à ce niveau ne présente pas les caractéristiques du bois de compression ; cette étude de LARSON, qui est la dernière consacrée à ce problème, montre donc que deux critères distinctifs du bois de compression considérés généralement comme indissociables : l'exagération de la croissance radiale et l'élévation de la densité du bois peuvent, fort bien, dans des circonstances déterminées, ne pas se manifester simultanément.

\section{II. - ORIENTATION GÉNÉRALE DE L'ANISOTROPIE, EXCENTRICITÉ MAXIMALE ET MÉPLAT}

Dans le cas particulier du Pin maritime des Landes, il importait de vérifier au départ l'orientation générale de l'anisotropie, qui est réputée se produire dans le sens ouest-est : l'étude en a été faite uniquement sur l'excentricité et le méplat, mais non sur la densité du bois.

L'excentricité de la moelle est définie par le rapport - multiplié par 100 du plus grand rayon au petit qui lui est opposé (1).

L'orientement de l'excentricité, mesuré en grades par rapport au nord magnétique, est celui du plus grand rayon. Une difficulté s'est présentée pour caractériser un orientement moyen à partir de mesures faites sur plusieurs arbres, puisque la moyenne de plusieurs orientements quelconques est en principe indéterminée. Mais quand les points figuratifs sur le cercle des orientements sont groupés, on peut néanmoins donner une valeur moyenne pour le groupe, à condition que l'origine des mesures ne soit pas située dans le groupe.

Le méplat d'une section est le rapport du plus grand axe absolu (ou diamètre) au plus grand axe de la direction perpendiculaire. L'orientement du méplat est celui du grand axe, mesuré dans le sens de l'excentricité.

(1) Le plus petit rayon est plus difficile à définir, car a apparaìt, surajoutée à l'anisotropie Est-Ouest, une anisotropie Sud-Nord, due probablement à la lumière (cf. Parrot 1960) mais qui est faible (sur 25 arores de l'Hermitage, les cernes Sud ne sont en moyenne que $8 \%$ plus larges que ceux du Nord); la conséquence en est que le plus petit rayon est, en général, orienté dans une direction Nord-Ouest, alors que le plus grand l'est a l'Est. 
Les différents résultats donnés ci-après ont été obtenus par mesures, soit sur des photographies de souches, soit sur des rondelles de 3 à $5 \mathrm{~cm}$ d'épaisseur prélevées de $2 \mathrm{~m}$ en $2 \mathrm{~m}$ sur des arbres abattus pour cette étude.

\section{1. - EXCENTRICITÉ DE SOUCHES D'ARBRES AGÉs DANS L'EST DU MASSIF LANDAIS}

Dans deux peuplements de 70 ans environ situés à Escaudes (Gironde) et Allons (Lot et Garonne), c'est-à-dire à une centaine de kilomètres de la mer, l'excentricité moyenne mesurée à la souche était respectivement de 143 et de 140 , pour 43 et 25 arbres, les orientements moyens étant 90 et 78 grades, soit assez près de la direction Est. Sur les 68 arbres, 10 étaient anisotropes vers l'Ouest, en un groupe nettement différent des autres (1). L'excentricité dans ce cas est de 106 (Escaudes, 4 arbres) et de 137 (Allons, 6 arbres).

\section{2. - ARBRES DE LA DUNE DU hUGA. CONSERVATION DE L'ANISOTROPIE LE LONG DE LA TIGE}

A l'opposé de ces arbres, à seulement $2 \mathrm{~km}$ de la mer, d'autres mesures ont été faites sur 13 arbres de forêts de dune, âgés d'environ 72 ans. Avant d'abattre les arbres, on a mesuré l'angle $\alpha$ qu'ils faisaient avec la verticale et l'orientement $\beta$ de la direction vers laquelle ils penchaient. L'angle $\alpha$ a été déterminé par son sinus, égal au rapport de la distance entre le point de projection au sol du niveau du premier verticille de branches vivantes et l'axe de la souche à la longueur de tige entre le sol et le premier verticille.

Les résultats sont indiqués dans le tableau 1 où sont portées les valeurs de l'excentricité et du méplat, ainsi que les orientements correspondants, pour les niveaux de $4 \mathrm{~m}, 6 \mathrm{~m}, 8 \mathrm{~m}$ et $10 \mathrm{~m}$ (les deux premières rondelles à 0 et $2 \mathrm{~m}$ n'ont pu être utilisées en raison des cares de gemmage).

Ici encore l'anisotropie maximale se présente en gros dans la direction Est-Ouest, en moyenne vers $130 \mathrm{gr}$, aussi bien pour l'excentricité que pour le méplat qui sont orientés dans la même direction. En raison du faible nombre d'arbres, on ne peut calculer des coefficients de corrélation entre les différents facteurs et, en particulier, entre l'intensité de l'inclinaison et l'anisotropie. Mais on observe une conservation de l'anisotropie le long de la tige, puisque les valeurs à $10 \mathrm{~m}$ sont aussi élevées qu'à $4 \mathrm{~m}$, de l'ordre de 125 pour l'excentricité et de 105 pour le méplat. Une estimation grossière au niveau de la souche montre que l'excentricité n'y est pas plus importante, et reste par conséquent très inférieure à celle des arbres de l'Est. Cela tient sans doute à ce que les 13 arbres du Huga sont situés sur le versant Est d'une dune, donc relativement abrités des forts vents de mer de l'Ouest.

(1) Ceci s'est produit aussi dans les autres cas étudiés plus loin : quand les arbres d'un peuplement sont anisotropes vers l'Ouest, ils forment un groupe très différent de leurs conpénères anisotropes vers l'Est. 
TABLEAU 1

Valeur de lanisotropie pour différents niveaux de $4 m$ à $10 m$ de 13 arbres adultes

Forêt domaniale de Lacanau, zone de dùne, 72 ans

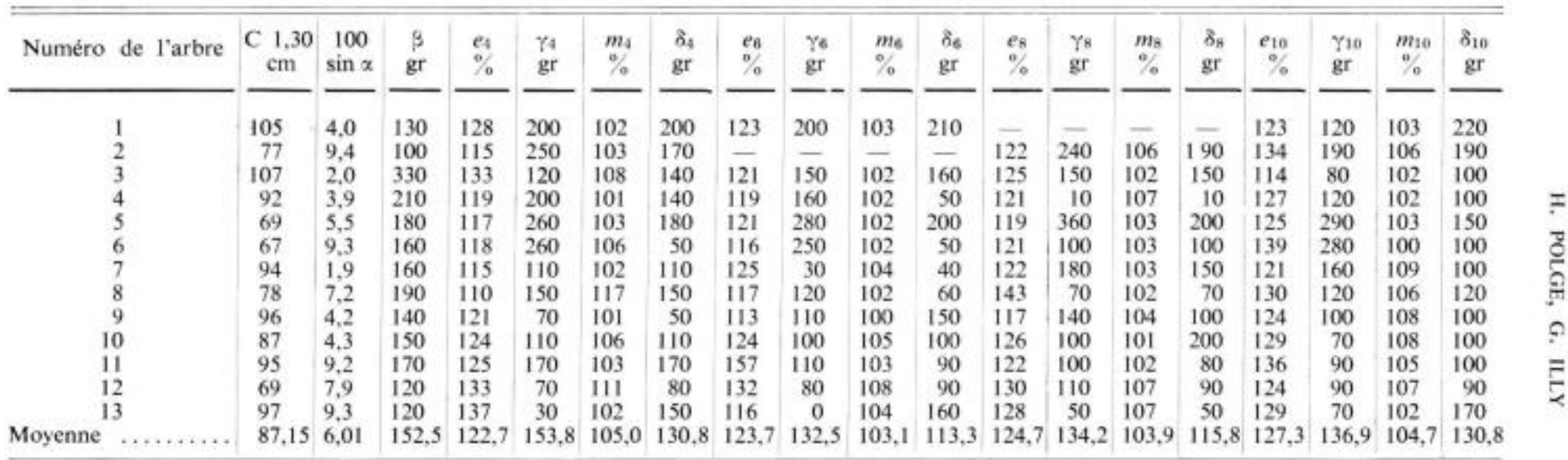

$\mathrm{C}=$ circonférence à $1,30 \mathrm{~m}$

$\alpha=$ angle que fait la tige avec la verticale

$\beta=$ orientation de l'inclinaison

$\gamma=$ orientation du plus grand rayon

$8=$ orientation du grand axe du méplat

$e=$ excentricité $=$ rapport du plus grand au plus petit rayon

$m=$ méplat $=$ rapport du plus grand axe absolu au plus grand axe perpendiculaire

Les indices $4,6,8$ et 10 se rapportent aux niveaux situés à $4 \mathrm{~m}, 6 \mathrm{~m}, 8 \mathrm{~m}$ et $10 \mathrm{~m}$. 
TABLEAU 2

Valeurs moyennes et corrélations entre les caractéristiques de 30 arbres et l'anisotropie à $2 \mathrm{~m}$ et à $4 \mathrm{~m}$

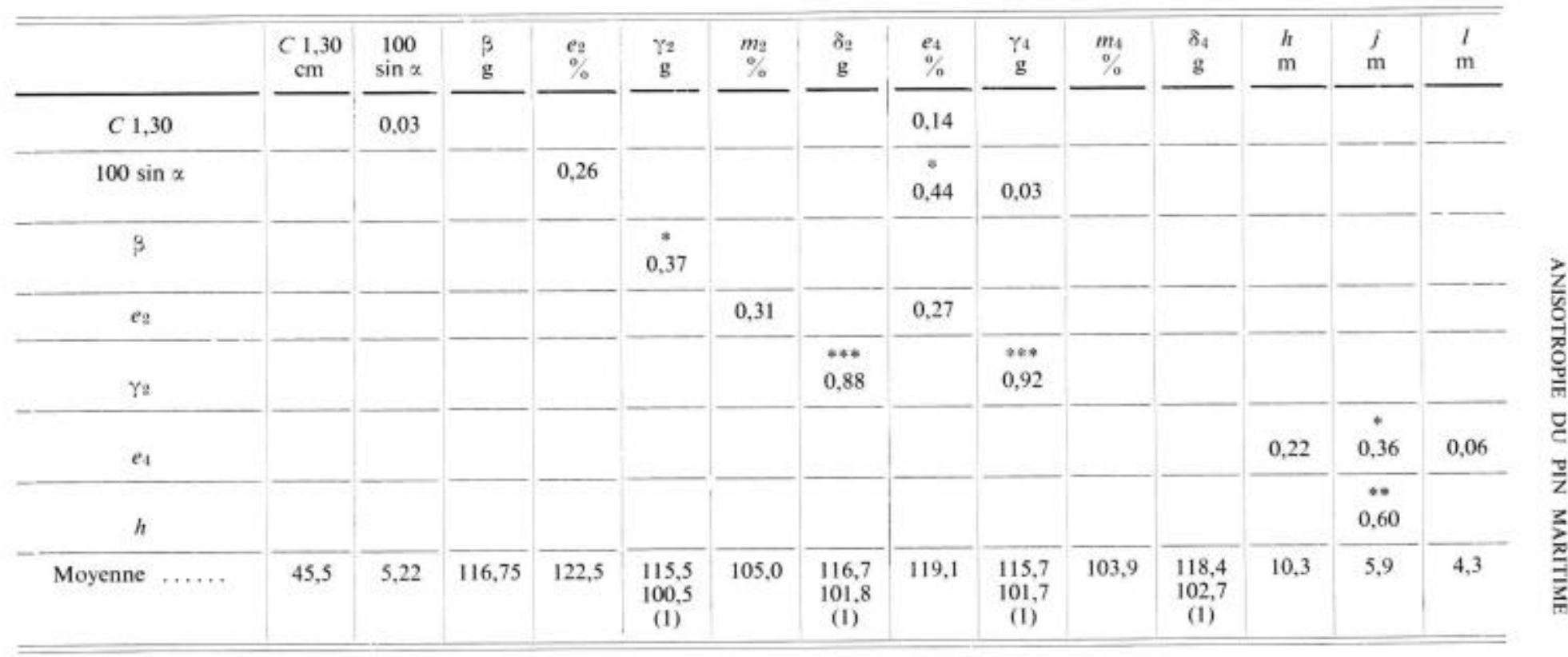

$C=$ circonférence à $1,30 \mathrm{~m}$

$x=$ angle que fait la tige avec la verticale

Seuils de signification

$3=$ orientation de l'inclinaison

$\gamma=$ orientation du plus grand rayon

$\delta=$ orientation du grand axe du méplat

$10 \%=0,31$

$5 \%=0,36^{*}$

$1 \%=0,46^{* *}$

$e=$ excentricité $=$ rapport du plus grand rayon au plus petit rayon

$1 \%=0,57^{* * *}$

$m=$ méplat $=$ rapport du plus grand axe absolu au plus grand axe perpendiculaire

$h=$ hauteur totale

$j=$ hauteur du premier verticille vert

$l=$ hauteur de cime $=h-j$

(1) La $2^{*}$ valeur moyenne pour $\gamma_{2}, \gamma_{4}, \delta_{2}, \delta_{4}$ est calculée sur 28 arbres seulement, les deux autres sont excentrés vers l'Est. 


\section{3. - ARBres de la pARCELle M DE L'Hermitage}

Sur 30 arbres de la parcelle $M$ de la forêt de l'Hermitage, âgés de 20 ans, on a mesuré, en même temps que l'excentricité et le méplat en valeur et en orientation à $2 \mathrm{~m}$ et à $4 \mathrm{~m}$, la hauteur de l'arbre, la hauteur du premier verticille vivant, la longueur de la cime et la circonférence à $1,30 \mathrm{~m}$ (tableau 2).

Les valeurs moyennes calculées sont du même ordre de grandeur que pour le Huga. L'inclinaison est voisine de $5 \%$, et le sens de l'inclinaison (fig. 1) ainsi que celui de l'anisotropie sont très sensiblement Est-Ouest, soit environ $100 \mathrm{gr}$. Des corrélations ont été calculées, dont certaines seront discutées plus loin (paragraphe 4) pour un essai d'explication.

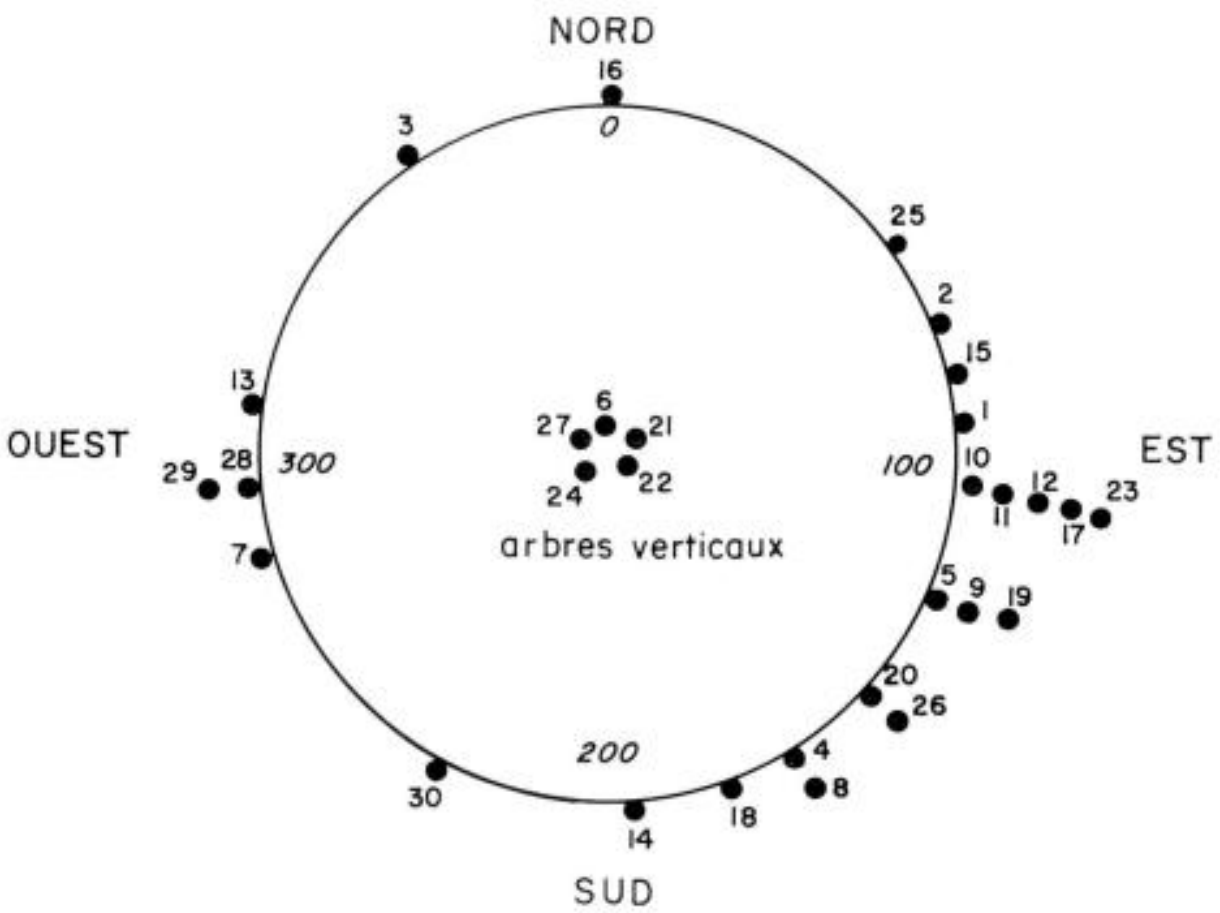

Fic. 1. - Orientation de Tinclinaison de 30 arbres de 19 ans. $L$ 'Hermitage, parcelle $M$.

La corrélation entre les orientations de l'inclinaison et de l'excentricité est certes significative, mais celles entre les orientations de l'excentricité à $2 \mathrm{~m}$ et à $4 \mathrm{~m}$, ou entre les orientations de l'excentricité et du méplat sont beaucoup plus élevées ; ceci confirme, d'une part que l'orientation de l'excentricité se conserve dans la tige, et d'autre part qu'excentricité et méplat sont dus aux mêmes causes. 


\section{III. - ANISOTROPIE EST-OUEST DU PIN MARITIME DES LANDES}

L'anisotropie Est-Ouest étant ainsi bien établie, on a procédé à de très nombreuses mesures sur des carottes de sondage extraites à la tarière de Pressler, qui ont évidemment le mérite de conserver vivants les arbres mesurés. Suivant les cas, l'anisotropie a été calculée de deux façons : ou bien on l'a estimée par le rapport de la valeur à 1'Est à celle de l'Ouest, et il s'agit d'une anisotropie relative Est-Ouest, ou bien on a fait le rapport de la plus grande à la plus petite des valeurs Est-Ouest, et il s'agit d'une anisotropie absolue Est-Ouest.

\section{1. - DENSITÉ ET LARGEUR DE CERNES}

\subsection{Observations faites sur des semis}

Dans une plantation comparative de descendances (référence D 440, cf. ILLY, 1966, Polge et ILly, 1967), les résultats moyens suivants ont été obtenus sur des semis de 4 ans (1) :

\section{TABLEAU 3}

\begin{tabular}{|c|c|c|c|c|c|c|c|}
\hline & \multirow{3}{*}{$\begin{array}{l}\text { Nombre } \\
\text { d'échan- } \\
\text { tillons }\end{array}$} & \multicolumn{2}{|c|}{ Densité moyenne } & \multirow{3}{*}{$\begin{array}{l}\text { Largeur } \\
\text { cerne } 65 \\
(\mathrm{en} \mathrm{cm})\end{array}$} & \multicolumn{3}{|c|}{ Excentricité relative } \\
\hline & & \multirow{2}{*}{$\begin{array}{l}\text { Totale } \\
\left(\mathrm{g} / \mathrm{dm}^{3}\right)\end{array}$} & \multirow{2}{*}{$\begin{array}{l}\text { Cerne } 65 \\
\left(\mathrm{~g} / \mathrm{dm}^{3}\right)\end{array}$} & & \multicolumn{2}{|c|}{ Densité moyenne } & \multirow{2}{*}{$\begin{array}{l}\text { Largeur } \\
\text { cerne } 65\end{array}$} \\
\hline & & & & & Totale & Cerne 65 & \\
\hline Côté Est & 237 & 331 & 326 & 1,23 & & & \\
\hline Côté Ouest & 237 & 319 & 313 & 1,13 & 103,8 & 104,2 & 108,8 \\
\hline
\end{tabular}

Ce test portait sur 9 familles différentes et, pour toutes les familles sans aucune exception, la densité était plus forte et les cernes plus larges à l'Est qu'à l'Ouest, l'écart maximum pour la moyenne d'une descendance se chiffrant en valeur relative à $7 \%$ pour la densité, et à $10 \%$ pour la largeur des accroissements annuels. Par contre, les comparaisons qui ont été faites sur la densité des branches principales d'orientation Est et Ouest sur le premier verticille n'ont fait apparaître entre elles aucune différence (la densité moyenne est de $436 \mathrm{~g} / \mathrm{dm}^{3}$ dans les deux cas).

Des précisions supplémentaires seront données au paragraphe 5 .

\subsection{Observations faites sur des arbres adultes}

\subsection{Arbres de la Forêt de I'Hermitage (Parcelle A).}

Sur 20 arbres âgés d'une cinquantaine d'années et maintenus sur pied après la coupe définitive du peuplement dont ils faisaient partie, ont été analysés, sur échan-

(1) Les densités indiquées icî, ainsi d'ailleurs que toutes celles dont il sera fait mention par la suite, sont des infra-densités (rapport du poids anhydre au volume saturé), après extraction de la résine par un mélange alcool-benzène. 
tillons prélevés à la tarière à différentes hauteurs, de $2 \mathrm{~m}$ en $2 \mathrm{~m}$, les variations de la densité du bois et des largeurs de cernes aux deux orientements Est et Ouest ; pour la densité, l'étude a été faite sur des tranches de 5 cernes numérotées à partir de la moelle, étant donné que, toutes choses égales par ailleurs, c'est le vieillissement de l'assise génératrice qui paraît conditionner la densité du bois élaboré ; pour les largeurs de cernes au contraire, ont été comparés des groupes de 2 ou 3 cernes produits durant les mêmes années, de façon à supprimer la principale cause de variabilité qui, pour les accroissements, est constituée par les changements de conditions climatiques d'une année à l'autre.

\section{- Variations globales de la densité du bois.}

Elles sont reportées de façon schématique sur la figure 2 : huit classes de densité ont été retenues, s'échelonnant de 300 à $500 \mathrm{~g} / \mathrm{dm}^{3}$, et représentées par un quadrillage conventionnel d'autant plus foncé que la densité est plus élevée ; les cases laissées en blanc ne traduisent pas une densité particulièrement faible, mais signifient simplement que le nombre d'échantillons obtenus était trop limité pour que les moyennes de densité les concernant puissent être considérées comme statistiquement valables ; d'assez nombreux prélèvements ne passaient pas exactement par la moelle des arbres en cause, et ceci explique que les cernes centraux se soient trouvés insuffisamment représentés à certains niveaux.

On constate tout d'abord, ce qui ne fait que confirmer les tendances générales de tous les arbres résineux, que la densité du bois, d'une part diminue avec la hauteur, d'autre part augmente avec la distance à la moelle ; mais on voit surtout qu'à tous les niveaux, et pour toutes les tranches de cernes, les densités moyennes Est sont, au moins égales, et, la plupart du temps, supérieures à celles de l'Ouest.

L'anisotropie moyenne de la densité a d'ailleurs été calculée aux différentes hauteurs, et les chiffres suivants ont été obtenus :

TABLEAU 4

\begin{tabular}{|c|c|c|c|c|c|c|c|c|c|c|}
\hline Hauteur (en m) & 0,30 & 1,30 & 3,30 & 5,30 & 7,30 & 9,30 & 11,30 & 13,30 & 15,30 & 17,30 \\
\hline $\begin{array}{l}\text { Anisotropic relative } \\
\text { Est-Ouest de la den- } \\
\text { sité }\end{array}$ & 105 & 105 & 109 & 110 & 106 & 107 & 108 & 106 & 106 & 109 \\
\hline
\end{tabular}

On voit que, si l'anisotropie semble légèrement plus faible aux niveaux inférieurs, il ne parait cependant pas possible de mettre en évidence une tendance générale de variation en fonction de la hauteur.

On peut mentionner accessoirement ici que les densités Nord et Sud ont ćgalement été analysées dans le cadre de la présente étude : aucune anisotropie parasite ne se manifeste dans cette direction, puisque, par exemple à $3,30 \mathrm{~m}$, les densités moyennes calculées sur 29 arbres sont, en g/dm ${ }^{3}$, de 417 au Sud, 417 à l'Ouest, 419 au Nord et 439 à l'Est, ce qui tend bien à prouver que c'est le bois de l'Est qui est anormalement dense, et non celui de l'Ouest anormalement léger. 


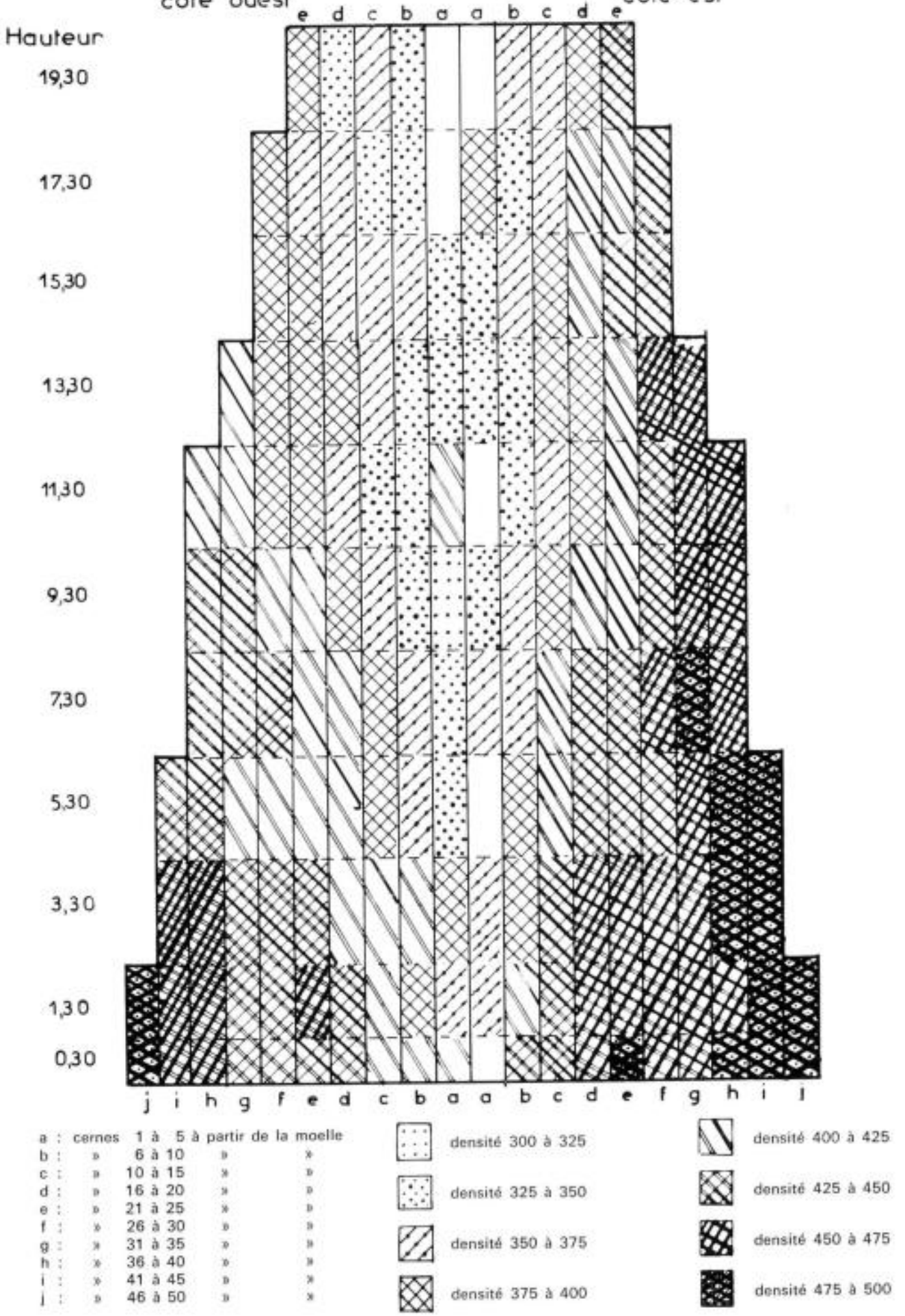

FIG. 2. - Variations de la densité du bois de pin maritime en fonction de la hauteur de Forientation et de la distance à la moelle. Moyenne's obtenues sur 20 arbres de la forêt de l'Hermitage, 
- Variations des largeurs de cernes

Sur la figure 3 sont représentées schématiquement à l'échelle les largeurs cumulées cies divers groupes d'accroissements annuels en cause aux différents niveaux. Là encore on peut observer que, sauf exceptions rarissimes, les accroissements Est sont supérieurs à ceux de l'Ouest, quelles que soient les années et les hauteurs considérées.

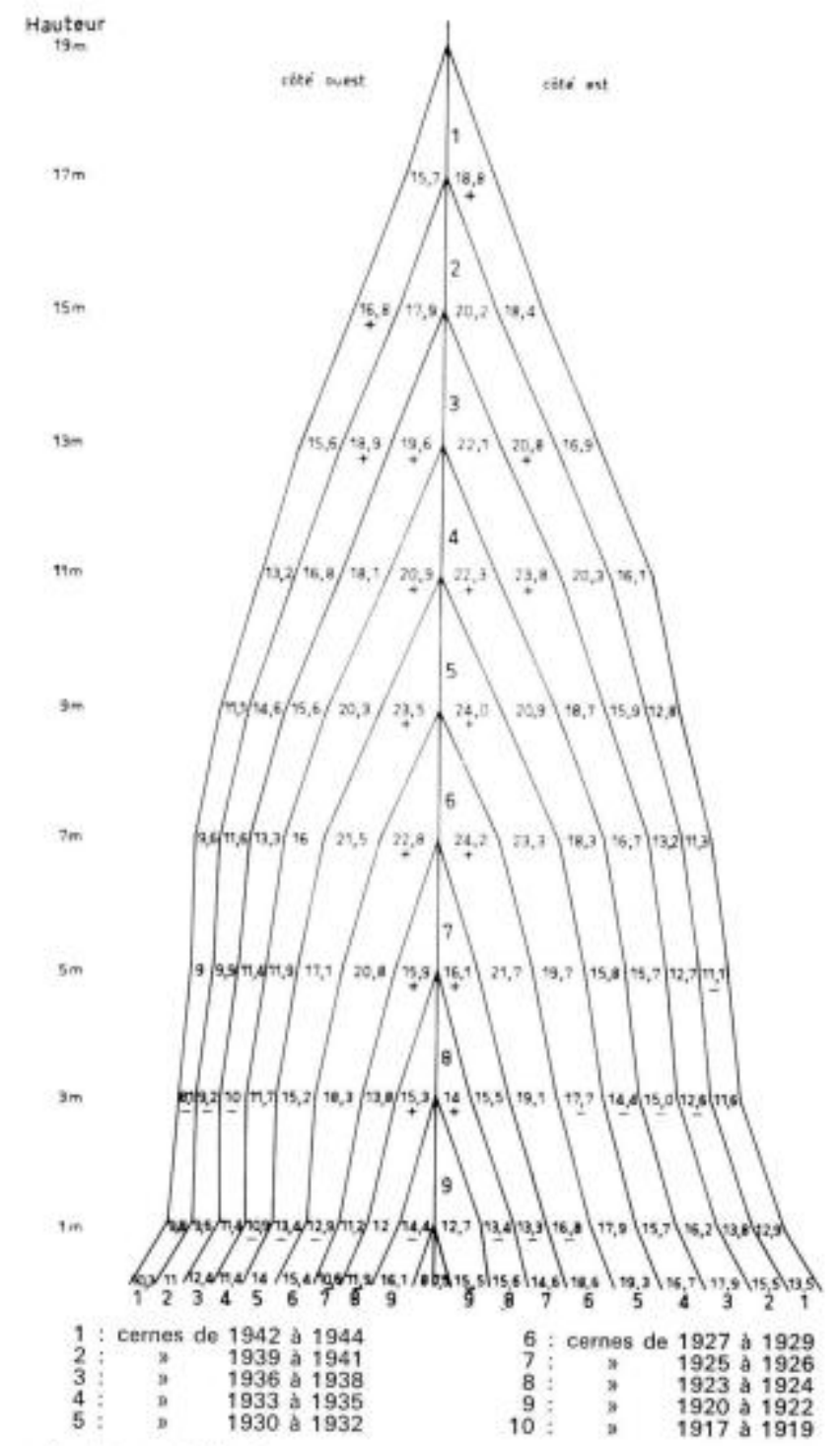

F.G. 3. - Variations de la largeur des cernes du pin maritime en fonction de la hauteur, de lorientation et de la distance à l'écorce (Moyennes obtemues sur 20 arbres en forêt de I'Hermitage) 
De plus ont été soulignés à l'aide d'une croix les niveaux auxquels ont été atteintes les largeurs de cernes maximales pour les diverses tranches annuelles, et par un trait simple les largeurs minimales. On constate que le niveau des accroissements les plus larges augmente avec l'âge et se situe presque toujours à la hauteur la plus élevée où ces cernes sont représentés, ce qui concorde bien avec la théorie généralement admise suivant laquelle les anneaux les plus épais se trouvent aux environs de la base de la partie vivante de la cime ; quant aux cernes de largeur minimale, on les retrouve aussi à des niveaux de plus en plus élevés au fur et à mesure que l'arbre vieillit, alors que l'on aurait pu s'attendre, au contraire, à les voir se maintenir aux niveaux inférieurs, étant donné le sens basipète de circulation de la sève ćlaborée et des substances de croissance.

\subsection{Ensemble des arbres plus et des témoins de la forêt landaise}

Les densités Est et Ouest ont été déterminées sur tous les arbres sélectionnés dans le cadre des recherches sur l'amélioration génétique du Pin maritime et sur les voisins auxquels ils sont comparés ; l'ensemble constitue un échantillonnage extrêmement valable puisque ces arbres se répartissent dans le massif landais à des latitudes et des distances de la mer très variables.

Pour 278 arbres, la densité moyenne générale est de $440 \mathrm{~g} / \mathrm{dm}^{3}$ à l'Est, et 416 seulement à l'Ouest, ce qui traduit une différence très significative au sens statistique du terme.

\section{2. - VARIATIONS CONTINUES DE DENSITÉ EN SECTION TRANSVERSALE}

Sur une carotte de sondage en provenance de l'Arboretum des Arrouilles passant rigoureusement par la moelle, ont été étudiées les variations de la densité par analyse densitomètrique de radiographie, suivant une méthode déjà décrite par ailleurs (Polge, 1966).

Les profils de densité correspondants, sur lesquels ont été reportées les années de production des divers accroissements, font l'objet de la figure 4 ; une analyse de variance a été faite portant sur les cinq caractéristiques suivantes : densité maximale annuelle, densité minimale annuelle, largeur des cernes, largeur du bois d'été et pourcentage du bois d'été, dont les valeurs moyennes étaient les suivantes :

TABLEAU 5

\begin{tabular}{|c|c|c|}
\hline & Côté Est & Côté Ouest \\
\hline Densités maximales annuelles. & 715,5 & 705,8 \\
\hline Densités minimales annuelles & 366,6 & 361,8 \\
\hline Largeur des cernes $(\mathrm{mm})$ & 3,46 & 2,68 \\
\hline 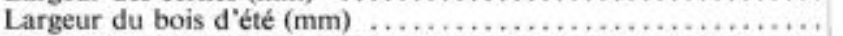 & 1,10 & 0,70 \\
\hline 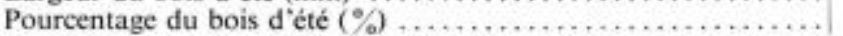 & 35,57 & 32,48 \\
\hline
\end{tabular}

Lorsqu'on prend comme source de variations les années, on trouve des différences très significatives pour les 5 caractéristiques en cause ; par contre, lorsqu'on 


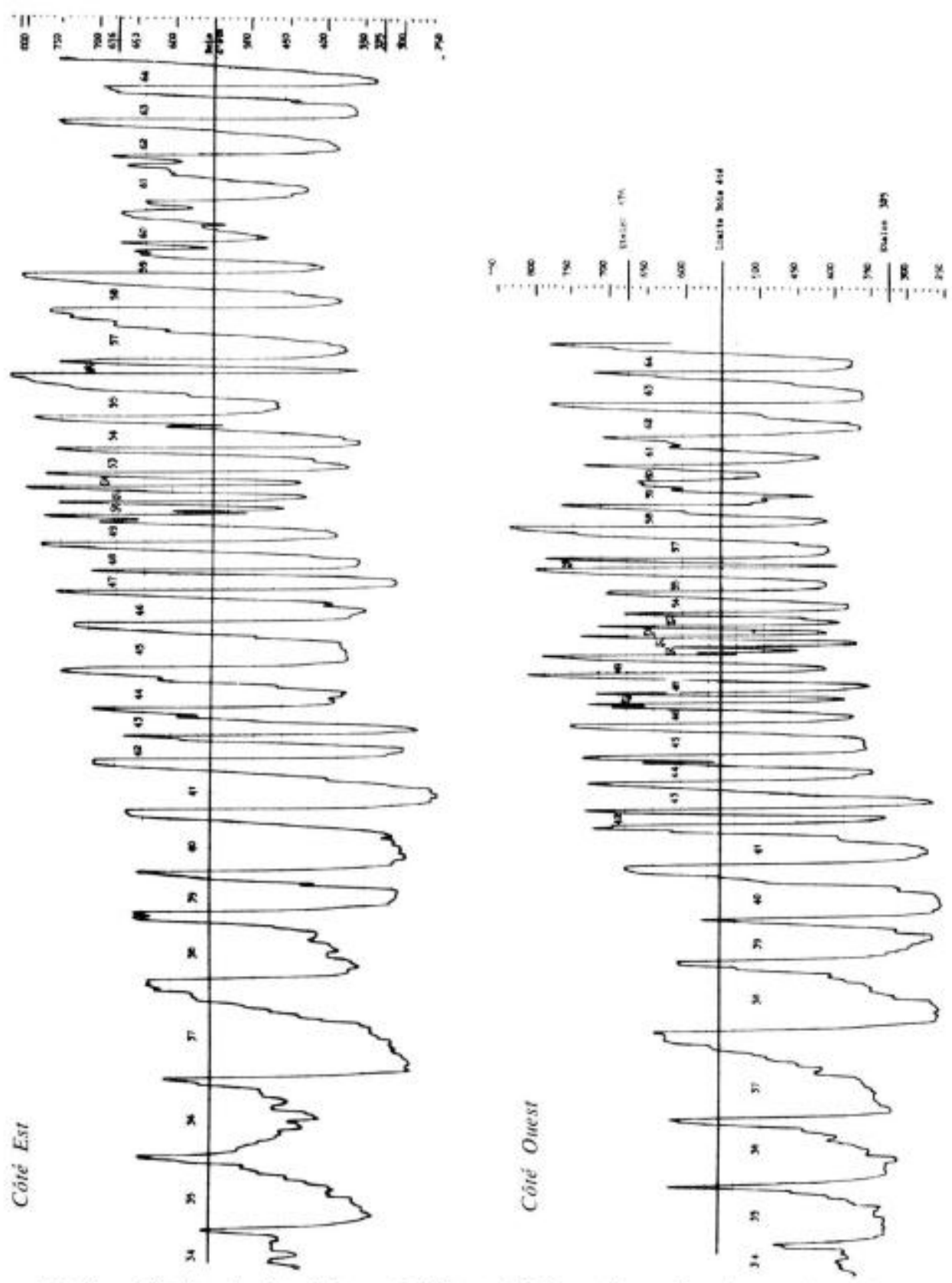

FIG. 4. - Courhes densitomêtriques cốté Est et cốté Ouest d'une même tige en pin maritime :

Enregistrement $n^{\circ} 739$

Objectif 4

Fente 130

Sensibilité 3.5

Densité 1 
étudie l'effet position, on observe qu'il n'y a aucune différence significative entre les côtés Est et Ouest pour les densités maximales, les densités minimales et les pourcentages de bois d'été, alors que les différences sont significatives, au seuil de $1 \%$, pour les largeurs de cernes et les largeurs de bois d'été.

On voit donc que, si le passage progressif du bois de printemps au bois d'été, qui est censé caractériser le bois de compression, n'est pas très apparent en ce qui concerne le Pin maritime des Landes, par contre, un nouveau critère, à savoir la largeur de bois d'été, apparaît comme particulièrement discriminant pour différencier les faces Est et Ouest des arbres de la forêt landaise.

On doit cependant signaler que des études analogues faites sur d'autres individus, aussi caractéristiques au point de vue anisotropie, ont conduit à de tout autres résultats en ce sens que, pour eux, existaient entre Est et Ouest des différences très significatives entre les densités minimales annuelles, qui étaient beaucoup plus élevées pour le côté Est que pour le côté Ouest.

\section{3. - DURETÉ}

La dureté des arbres sur pied a été estimée dans un certain nombre de placeaux de l'Arboretum des Arrouilles (1) sous forme du couple de torsion nécessaire pour enfoncer la tarière à une profondeur de $2,8 \mathrm{~cm}$ sous écorce ; cette détermination a été faite à l'Est et à l'Ouest et a donné les résultats suivants :

TABLEAU 6

\begin{tabular}{|c|c|c|}
\hline \multirow{2}{*}{ No du placeau } & \multicolumn{2}{|c|}{ Couple de torsion (en $\mathrm{cm}, \mathrm{kg}$ ) } \\
\hline & EST & OUEST \\
\hline 1 & 250 & 225 \\
\hline 2 & 287 & 235 \\
\hline 3 & 275 & 235 \\
\hline 5 & 234 & 191 \\
\hline 6 & 237 & 213 \\
\hline 7 & 230 & 205 \\
\hline 8 & 252 & 217 \\
\hline 9 & 305 & 257 \\
\hline 10 & 287 & 250 \\
\hline 11 & 231 & 215 \\
\hline 13 & 278 & 237 \\
\hline 16 & 260 & 233 \\
\hline 17 & 273 & 243 \\
\hline 19 & 221 & 189 \\
\hline 20 & 242 & 200 \\
\hline 22 & 268 & 232 \\
\hline 23 & 227 & 207 \\
\hline 29 & 220 & 218 \\
\hline Total $\ldots \ldots \ldots \ldots \ldots \ldots \ldots \ldots$ & 4577 & 4002 \\
\hline Moyenne .................... & 254 & 222 \\
\hline
\end{tabular}


On trouve donc une forte anisotropie au point de vue dureté, ce qui ne doit pas surprendre puisqu'une corrélation étroite existe entre les couples de torsion ainsi mesurés et la densité du bois.

\section{4. - RÉTRACTIBILITÉ}

\subsection{Retrait longitudinal}

Sur un arbre dont l'anisotropie était particulièrement marquée, des éprouvettes d'essais de rétractibilité axiale ont été confectionnées, et les résultats moyens obtenus pour divers accroissements annuels ont été les suivants $(\%$ de retrait entre l'état saturé et l'état sec à l'air) :

TABLEAU 7

\begin{tabular}{|c|c|c|c|}
\hline & & EST & OUEST \\
\hline $\begin{array}{l}1953 \\
1957 \\
1963\end{array}$ & 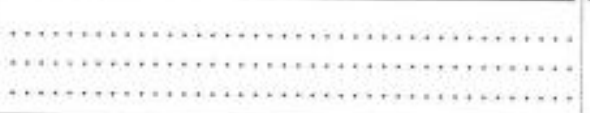 & $\begin{array}{l}0,26 \\
0,35 \\
0,32\end{array}$ & $\begin{array}{l}0,03 \\
0,05 \\
0,1\end{array}$ \\
\hline
\end{tabular}

On voit que, si la rétractibilité du côté Est est toujours supérieure à celle de l'Ouest, elle demeure cependant très faible et sans conséquence grave au point de vue technologique, n'atteignant que le dixième environ de la rétractibilité longitudinale du vrai bois de compression.

\subsection{Retrait transversal}

Le bois de compression s'apparentant par beaucoup de côtés au bois juvénile, les retraits tangentiel et radial ont été déterminés sur 4 séries d'éprouvettes différentes : des éprouvettes Est en bois adulte et en bois juvénile, des éprouvettes Ouest, également en bois adulte et en bois juvénile ; le tableau ci-dessous donne les résultats moyens pour 20 éprouvettes dans chaque série.

TABLEAU 8

\begin{tabular}{|c|c|c|c|c|c|}
\hline & & \multicolumn{2}{|c|}{ Côté EST } & \multicolumn{2}{|c|}{ Côté OUEST } \\
\hline & & $\begin{array}{l}\text { Bois } \\
\text { adulte }\end{array}$ & $\begin{array}{l}\text { Bois } \\
\text { juvénile }\end{array}$ & $\begin{array}{l}\text { Bois } \\
\text { adulte }\end{array}$ & $\begin{array}{c}\text { Bois } \\
\text { juvénile }\end{array}$ \\
\hline \multirow{2}{*}{$\begin{array}{l}\text { Retrait } \\
\text { tangentiel } \\
\text { en } \%\end{array}$} & f Saturé à anhydre $\ldots . .$. & 3,78 & 3,37 & 4,74 & 3,99 \\
\hline & ' Sec à anhydre .... & 2,85 & 2,24 & 3,05 & 2,36 \\
\hline \multirow{2}{*}{$\begin{array}{l}\text { Retrait } \\
\text { radial en \% }\end{array}$} & S Saturé à anhydre $\ldots$ & 2,21 & 1,71 & 2,74 & 1,81 \\
\hline & I Sec à anhydre.... . & 1,51 & 1,12 & 1,60 & 1,41 \\
\hline
\end{tabular}


Aussi bien pour le retrait tangentiel que pour le retrait radial, et aussi bien entre l'état saturé et l'état anhydre qu'entre l'état sec à l'air et l'état anhydre, les retraits transversaux sont plus faibles à l'Est qu'à l'Ouest, et plus faibles pour le bois juvénile que pour le bois adulte. On doit rappeler à ce sujet qu'un retrait tangentiel plus faible est signalé dans la littérature comme caractérisant le bois de compression : mais aucune référence n'a malheureusement pu être trouvée en ce qui concerne le retrait radial.

\section{5. - CARACTÉRISTIQUES ANATOMIQUES}

De nombreuses coupes microscopiques ont été réalisées dans divers échantillons de Pin maritime des Landes, côté Est et côté Ouest ; on peut dire que, de ce point de vue, le bois du côté Est présente, mais sous une forme atténuée, certaines caractéristiques anatomiques du bois de compression : les trachéides y sont légèrement plus arrondies que celles du bois normal, et l'on peut observer quelquefois, mais rarement, la présence de petits méats intercellulaires.

La figure 5 concerne l'échantillon qui a paru se rapprocher le plus du bois de compression typique ; il s'agit donc d'un cas extrême, et pourtant, fibres arrondies et méats intercellulaires ne se retrouvent même pas sur la totalité de la coupe ; cepen-

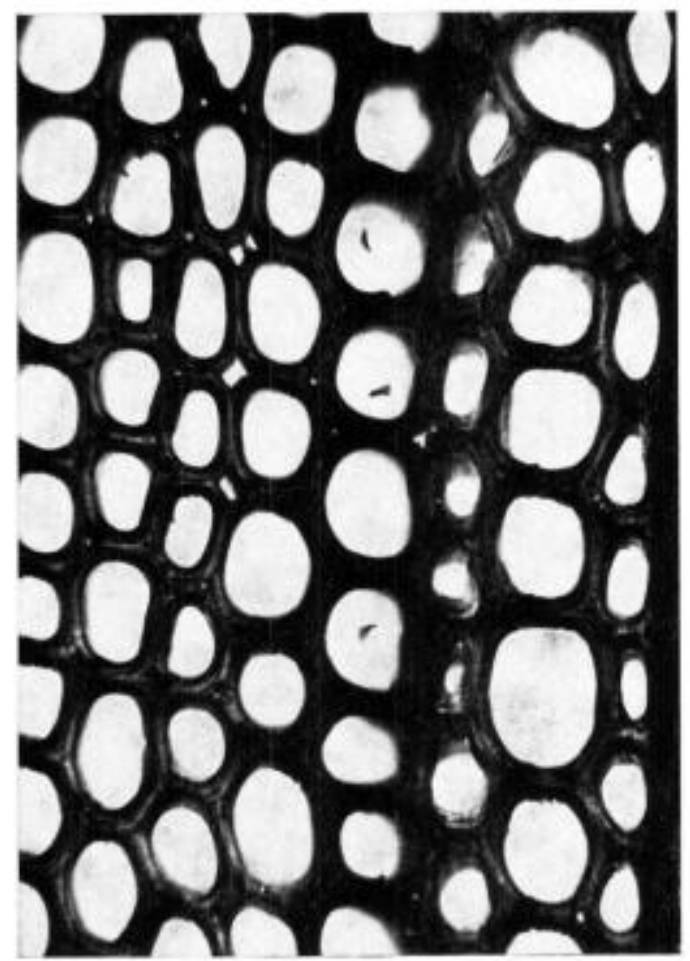

FIG, 5, - Coupe microscopique dans du bois de l'Est présentant certaines caractéristiques du bois de compression (grossissement environ 400) 
dant, dans le même échantillon examiné en section tangentielle, apparaissent bien les stries en spirales du bois de compression avec une inclinaison qui peut atteindre $30^{\circ}$ par rapport à l'axe de fibre.

\section{6. - CARACTÉRISTIQUES PAPETIĖRES}

Le rendement en fibres a été déterminé, et la longueur des fibres mesurée, sur un échantillonnage de 100 fibres dans chaque cas, pour deux accroissements annuels sur le côté Est et sur le côté Ouest ; les résultats ont été les suivants :

TABLEAU 9

\begin{tabular}{|c|c|c|c|c|}
\hline & \multicolumn{2}{|c|}{1955} & \multicolumn{2}{|c|}{1960} \\
\hline & EST & OUEST & EST & OUEST \\
\hline Rendement en fibres $(\%), \ldots \ldots \ldots \ldots \ldots \ldots . . . . .$. & 44 & 50 & 45 & 53 \\
\hline Longueur moyenne des fibres $(\mathrm{mm}) \ldots \ldots \ldots \ldots \ldots$ & 1,90 & 2,33 & 2,46 & 2,70 \\
\hline
\end{tabular}

Quelle que soit l'année en cause, le rendement en fibres et la longueur moyenne des fibres sont moins élevés du côté Est que du côté Ouest ; pour la longueur, on retrouve donc à nouveau, sur le côté Est, une caractéristique reconnue du bois de compression, cependant que la baisse du rendement observée sur ce même côté peut être rapprochée de la teneur en lignine plus élevée qui, d’après la littérature, est également un critère distinctif du bois de compression.

\section{IV. - ÉTUdE DES CAUSES POSSIBLES DE L'ANISOTROPIE DU PIN DES LANDES}

\section{1. - INFLUENCE DE L'INCLINAISON DES TIGES}

On a vu que, pour des résineux à tronc non vertical, du bois de compression devrait se former du côté vers lequel penchent les arbres. Or l'étude des arbres âgés de la parcelle $A$ de I'Hermitage n'a rien montré de significatif à ce sujet.

Par contre, pour ceux de la parcelle $M$ (tableau 2), l'étude des corrélations est intéressante. On y voit en effet que l'excentricité est liée à l'inclinaison de l'arbre, mais de façon significative seulement à $4 \mathrm{~m}$, et non à $2 \mathrm{~m}$ : ceci n'a rien d'étonnant car les arbres ont pu être inclinés au départ de façon différente, soit par héliotropisme, soit par un coup de vent. En déduire que l'excentricité est due à l'inclinaison serait hasardeux, les deux phénomènes pouvant être liés parce que dus tous deux à la même cause, c'est-à-dire l'action du vent sur l'arbre. Notons aussi que sur les 30 arbres, 5 sont verticaux (figure 1), mais 4 d'entre eux sont excentrés vers l'Est (c'està-dire la moelle vers l'Ouest), et que sur les 6 arbres inclinés vers l'Ouest, un seul est excentré vers l'Ouest. 


\section{2. - INFLUENCE DU VENT}

Dans le cadre du test D 440, on a essayé de voir si les vents dominants qui soufflent de l'Ouest pouvaient être considérés comme responsables de l'anisotropie des Pins de la forêt landaise ; à cet effet, cette anisotropie, aussi bien au point de vue densité qu'au point de vue largeur de cernes, a été calculée sur 116 plants répartis au hasard entre les diverses familles mais situés, les uns sur la lisière Ouest du dispositif exposée au vent, les autres sur la lisière Est, oủ l'on peut considérer que les plants étaient abrités par le reste de la parcelle d'expérience.

Les résultats moyens suivants ont été obtenus :

TABLEAU 10

\begin{tabular}{|c|c|c|c|c|c|c|c|}
\hline \multicolumn{4}{|c|}{ Lisière EST } & \multicolumn{4}{|c|}{ Lisière OUEST } \\
\hline \multicolumn{2}{|c|}{$\begin{array}{l}\text { Densité } \\
\text { en } \mathrm{g} / \mathrm{dm}^{3}\end{array}$} & \multicolumn{2}{|c|}{$\begin{array}{l}\text { Largeur de cernes } \\
\text { en } \mathrm{mm} \times 100\end{array}$} & \multicolumn{2}{|c|}{$\begin{array}{l}\text { Densité } \\
\text { en } \mathrm{g} / \mathrm{dm}^{3}\end{array}$} & \multicolumn{2}{|c|}{$\begin{array}{l}\text { Largeur de cernes } \\
\text { en } \mathrm{mm} \times 100\end{array}$} \\
\hline Est & Ouest & Est & Ouest & Est & Ouest & Est & Ouest \\
\hline 323,3 & 316,2 & 121,3 & 110,3 & 332,2 & 311,5 & 125,7 & 114,9 \\
\hline
\end{tabular}

Des tests de comparaison de moyennes portant sur les écarts entre caractéristiques Est et caractéristiques Ouest ont été faits ; ils ont montré qu 'il n'y a pas de différence significative entre les lisières Est et Ouest pour l'anisotropie des largeurs de cernes, mais qu'il existe par contre une différence significative au seuil de $5 \%$ pour l'anisotropie des densités, c'est-à-dire qu'il y a significativement plus de différence entre les côtés Est et Ouest pour les plants exposés aux vents dominants qui viennent de l'Océan que pour ceux qui sont protégés par le reste du placeau.

De même, on constate que, pour les arbres de la parcelle $M$ (tableau 2), la hauteur de l'arbre ou la longueur de la cime n'agissent pas sur l'excentricité, alors que la hauteur du premier verticille lui est liée ; on peut penser à deux " explications » de ce fait, l'une, mécanique (bras de levier plus long quand la cime commence plus haut), l'autre, hormonale (plus grande distance de diffusion de l'auxine).

\section{V. - ÉTUDES DES VARIATIONS HÉRÉdITAIRES}

\section{1. - VARIATIONS ENTRE PROVENANCES}

\subsection{Arbres de l'Arboretum des Arrouilles}

Cet arboretum a été créé pour comparer entre elles un certain nombre de provenances de Pins maritimes, et présente l'intérêt de remonter à 1926 pour les placeaux les plus anciens.

Les résultats moyens obtenus sur carottes prélevées à $1,30 \mathrm{~m}$ sont consignés dans le tableau 11. (Cf. ILLY, 1966). 
Il semble bien que les provenances landaises aient une anisotropie relativement plus élevée que la moyenne des autres et que, par contre, les provenances marocaines ou certaines provenances méditerranéennes se caractérisent par des différences assez faibles, soit de densité, soit de largeur de cernes, entre les faces Est et Ouest. Mais il existe des exceptions notables, comme celle du placeau $\mathrm{n}^{\circ} 3$ par exemple, dont les arbres sont dotés d'une anisotropie peu marquée, ou, en sens contraire, comme le placeau $n^{\circ} 20$, qui, parmi les provenances méditerranéennes, se singularise par une forte anisotropie, tout au moins en ce qui concerne la densité.

\section{TABLEAU 11}

Anisotropie de la densité el des largeurs de cernes pour differentes provenances (arboretum des Arrouilles)

\begin{tabular}{|c|c|c|c|c|c|c|c|c|}
\hline \multirow{2}{*}{$\begin{array}{l}\mathrm{N}- \\
\text { du } \\
\text { pla- } \\
\text { ceau }\end{array}$} & \multirow[b]{2}{*}{ Provenance } & \multirow[b]{2}{*}{$\begin{array}{c}\text { Nbre } \\
\text { d'arbres }\end{array}$} & \multicolumn{3}{|c|}{$\begin{array}{l}\text { Densité moyenne } \\
\quad\left(e n \mathrm{~g} / \mathrm{dm}^{3}\right)\end{array}$} & \multicolumn{3}{|c|}{$\begin{array}{l}\text { Largeur moyenne } \\
\text { de cernes (en } \mathrm{mm} \text { ) }\end{array}$} \\
\hline & & & Est & Ouest & $\begin{array}{l}\text { Aniso- } \\
\text { tropie } \\
\text { rela- } \\
\text { tive }\end{array}$ & Est & Ouest & $\begin{array}{c}\text { Aniso- } \\
\text { tropie } \\
\text { relati- } \\
\text { ye }\end{array}$ \\
\hline 1 & Seignosse (Sud Landes) ...... & II & 467 & 435 & 107,4 & 3,12 & 2,30 & 136 \\
\hline 2 & Vieille St-Girons (Sud Landes) ... & 12 & 479 & 447 & 107,2 & 3,44 & 2.75 & 125 \\
\hline 3 & La Teste (Sud Gironde) ........... & 13 & 460 & 439 & 104,8 & 3,47 & 2,82 & 123 \\
\hline 5 & La Teste (Sud Gironde) .... & 14 & 421 & 404 & 104,2 & 3,74 & 2,78 & 135 \\
\hline 6 & Lège et Garonne (Gironde) & 13 & 460 & 441 & 104,3 & 3,15 & 2,48 & 127 \\
\hline 7 & Lège et Garonne (Gironde) ........ & 15 & 446 & 431 & 103,5 & 2,77 & 2,05 & 135 \\
\hline 8 & St-Augustin (Charente-Maritime). & 14 & 457 & 422 & 108,3 & 2,56 & 2,06 & 124 \\
\hline 9 & St-Augustin (Charente Maritime).. & 13 & 480 & 465 & 105,4 & 2,89 & 2,31 & 125 \\
\hline 10 & Narbonne (Aude) ............. & 12 & 455 & 455 & 100 & 2,92 & 2,54 & 115 \\
\hline 11 & Ares (Maures) ...................... & 12 & 458 & 446 & 102,7 & 3,06 & 2,57 & 119 \\
\hline 12 & Braus (Alpes-Maritimes) . ......... & 10 & 424 & 413 & 102,7 & 3,47 & 2,90 & 120 \\
\hline 13 & Portugal $\ldots \ldots \ldots \ldots \ldots \ldots$ & 16 & 453 & 416 & 108,9 & 3,38 & 2,75 & 123 \\
\hline 16 & Chinon (reboisement) ............. & 12 & 442 & 423 & 104,5 & 2,87 & 2,38 & 121 \\
\hline 17 & 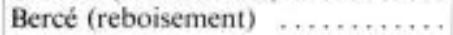 & 11 & 467 & 439 & 106,4 & 3,12 & 2,95 & 106 \\
\hline 19 & Esterel ...................... & 12 & 426 & 412 & 103,4 & 3,48 & 3,09 & 113 \\
\hline 20 & Esterel $\ldots \ldots \ldots \ldots \ldots \ldots \ldots \ldots \ldots \ldots$ & 13 & 431 & 396 & 108,8 & 3,35 & 2,96 & 113 \\
\hline 21 & Sospel (Alpes-Maritimes) ...... & 10 & 410 & 384 & 106,8 & 3,87 & 3,18 & 122 \\
\hline 22 & Campine Belge (reboisement) ..... & 14 & 430 & 420 & 102,4 & 3,05 & 2,68 & 114 \\
\hline 23 & Calenzana (Corse) $\ldots \ldots \ldots \ldots \ldots$ & 10 & 433 & 428 & 101,2 & 3,49 & 2,82 & 124 \\
\hline 24 & Calenzana (Corse) .................... & 6 & 392 & 386 & 101,5 & 2,60 & 2,29 & 114 \\
\hline 25 & Zonza Ouest (Corse) ................ & 10 & 455 & 423 & 107,6 & 2,55 & 2,20 & 116 \\
\hline 29 & Maroc-Aīn Leuh ..................... & 13 & 421 & 424 & 99,3 & 3,45 & 2,81 & 123 \\
\hline 30 & Maroc $\ldots \ldots \ldots \ldots \ldots \ldots$ & 19 & 399 & 392 & 101,8 & 2,76 & 2,40 & 115 \\
\hline \multirow[t]{2}{*}{31} & Maroc $\quad \ldots \ldots \ldots \ldots \ldots \ldots . . . \ldots \ldots$ & 20 & 383 & 374 & 102,4 & 2,27 & 1,99 & 114 \\
\hline & Moyennes & & 439,5 & 421,5 & 104,4 & 3,12 & 2,59 & 120,9 \\
\hline
\end{tabular}

Le cas du placeau 3 peut très bien s'expliquer par le fait qu'il s'agit, non pas d'une vraie provenance obtenue à partir de plusieurs semenciers, mais de la descendance d'un seul arbre qui pouvait très bien être particulier. Pour le placeau 20, on ne sait rien sur son origine, mais comme il a été établi en même temps que le placeau 19, la moyenne des deux placeaux peut être retenue. Dans l'ensemble, on peut donc 
considérer les provenances méditerranéennes, qui par ailleurs sont moins denses, comme moins anisotropes que les provenances landaises.

En ce qui concerne la dureté ( $\$ 3.3$ ), bien que le classement des diverses provenances ne soit pas identique à celui obtenu pour l'anisotropie de la densité, on peut observer qu'un certain nombre de placeaux, pour lesquels les différences de densité entre l'Est et l'Ouest sont faibles (placeaux $n^{08} 11,23$ et 29 par exemple), figurent également parmi ceux qui sont dotés d'une assez bonne homogénéité au point de vue dureté.

\subsection{Plantation comparative des Malgaches}

Dans cet essai plus jeune (les arbres mesurés avaient 15 ans), on a fait des mesures de l'anisotropie maximum et du méplat à 2 niveaux $(2 \mathrm{~m}$ et $4 \mathrm{~m}$ ) pour 3 provenances qui nécessitaient une éclaircie (tableau 12) : si la provenance Corse est nettement plus verticale, le méplat en est très peu différent de celui des 2 provenances landaises, et l'excentricité à peine inférieure en moyenne pour les différents niveaux.

TABLEAU 12

Anisotropie de diverses provenances (Plantation des Malgaches)

\begin{tabular}{|c|c|c|c|c|c|c|}
\hline $\begin{array}{c}\text { Caractéristique } \\
\text { Provenance }\end{array}$ & $\begin{array}{l}\text { Nombre } \\
\text { d'arbres }\end{array}$ & $\begin{array}{c}\text { Arbres } \\
\text { verticaux }\end{array}$ & $\begin{array}{l}\text { Excentrici- } \\
\text { té \% a } 2 \text { m }\end{array}$ & $\begin{array}{l}\text { Mèplat } \\
\text { à } 2 \mathrm{~m}\end{array}$ & $\begin{array}{l}\text { Excentri- } \\
\text { cité \%à } 4 \mathrm{~m}\end{array}$ & $\begin{array}{l}\text { Méplat } \\
\text { à } 4 \mathrm{~m}\end{array}$ \\
\hline $\begin{array}{l}\text { Landes-Bias } \ldots \ldots \ldots . . . \\
\text { Landes-Tremblade....... } \\
\text { Corse-Corté ........... }\end{array}$ & $\begin{array}{l}10 \\
20 \\
10\end{array}$ & $\begin{array}{l}0 \\
1 \\
7\end{array}$ & $\begin{array}{l}112,5 \\
118,6 \\
113,1\end{array}$ & $\begin{array}{l}104,9 \\
104,7 \\
103,6\end{array}$ & $\begin{array}{l}120,0 \\
117,0 \\
113,8\end{array}$ & $\begin{array}{l}102,1 \\
104,1 \\
102,8\end{array}$ \\
\hline
\end{tabular}

\section{2. - VARIATIONS INDIVIDUELLES DANS LA PROVENANCE LANDAISE}

Dans l'essai D 440, déjà cité, nous avons pu étudier, d'une part l'héritabilité, d'autre part les corrélations génétiques des différentes mesures concernant l'anisotropie (1).

\subsection{Héritabilités}

Pour les 237 plants de 9 familles de demi-frères nous avons calculé par la méthode de la décomposition de variances l'héritabilité de l'anisotropie Est-Ouest absolue, c'est-à-dire du rapport du plus grand au plus petit des rayons Est et Ouest : cette héritabilité est de $21 \%$, valeur significative, puisque le test $F$ du carré moyen des familles rapporté au carré moyen des individus dans les familles est significatif avec une probabilité comprise entre 0,05 et 0,01 . Mais la précision est très faible en raison du petit nombre de familles.

Ce résultat laisse cependant entrevoir la possibilité d'une sélection, positive ou négative suivant les désirs des utilisateurs, du caractère d'anisotropie sur la largeur de cernes.

(1) Il faut souligner que l'anisotropie, telle que nous l'awons définie par un rapport de grandeurs, a des chances sur le plan statistique de ne pas suivre une loi normale si les grandeurs sont elles-mèmes normales. Mais, vérification faite, l'hypothèse de normalité peut être admise ici pour l'anisotropie. 
Le même type de calculs appliqué aux anisotropies relatives $1=$ densité totale Est/densité totale Ouest, 2 = densité du cerne 1965 Est/densité du cerne 1965 Ouest et 3 = largeur du cerne 1965 Est/largeur du cerne 1965 Ouest a donné des tests $F$ non significatifs, et par conséquent n'a pas permis de calculer les héritabilités correspondantes.

\subsection{Corrélations entre caractères d'anisotropie}

On sait qu'on peut définir la corrélation phénotypique entre deux caractères pour des individus dont on ne tient pas compte de la parenté et qui est faite à la fois de la corrélation génétique entre ces caractères et de la corrélation due aux facteurs du milieu. La corrélation génétique peut se calculer quand on dispose de groupes de structure connue. La corrélation entre moyennes des familles dépend du nombre d'individus dans les familles mais se rapproche plus ou moins de la corrélation génétique : elle présente l'avantage de se calculer facilement sans l'intervention d'un ordinateur. La corrélation entre moyennes de provenances présente un intérêt similaire.

\subsection{Corrélations génétiques}

La connaissance des corrélations génétiques est très importante pour le sélectionneur pour savoir si l'action en faveur d'un caractère intéressant aura une répercussion favorable, défavorable ou nulle sur un autre caractère.

Les corrélations génétiques ont d'abord été calculées entre les valeurs de différentes grandeurs à l'Est et à l'Ouest et d'autres caractères des arbres de l'essai D 440 (cf. Polge et Illy, 1967).

TABLEAU 13

Corrélations gẻnériques

\begin{tabular}{|c|c|c|c|c|c|c|}
\hline \multicolumn{7}{|c|}{ Caractéristiques des arbres } \\
\hline & Forme & Hauteur & $\begin{array}{l}\text { Section } \\
\text { à mi-haut. }\end{array}$ & $\begin{array}{l}\text { Densité } \\
\text { à la base }\end{array}$ & $\begin{array}{c}\text { Section } \\
\text { cerne 1965. } \\
\text { EST }\end{array}$ & $\begin{array}{c}\text { Densité } \\
\text { cerne } 1965 \\
\text { OUEST }\end{array}$ \\
\hline Largeur cerne 1965-EST . & $-1,09$ & 1,30 & 1,08 & 1,14 & 0,55 & 0,99 \\
\hline $\begin{array}{l}\text { Largeur cerne } \\
\text { 1965-OUEST } . . . . . . .\end{array}$ & $-0,48$ & $-0,41$ & 0,72 & 0,20 & 0,56 & 1,14 \\
\hline
\end{tabular}

On voit ainsi que, si la largeur du cerne 1965 à l'Est est liée positivement à la section à la base, elle l'est négativement à la forme et à la hauteur, et ceci de façon beaucoup plus forte que celle du côté Ouest ; le côté Est qui représente le bois anormal est donc lié à des caractères défavorables, alors que les liaisons avec la section à mi-hauteur, caractère favorable, sont plus voisines entre elles.

On observe aussi que c'est la densité du cerne Ouest qui est la plus liée à la largeur du cerne aussi bien Est qu'Ouest. La densité Ouest pourrait ainsi servir à une sélection indirecte pour la croissance en diamètre si cette liaison existait, non seule- 
ment au niveau du cerne 1965, mais sur un ensemble important d'accroissements : nous n'avons pas encore pu le vérifier.

La corrélation génétique a, de même, été calculée pour les anisotropies relatives Est-Ouest citées au paragraphe 5.21 : elle est de 0,95 entre 1 et 2 (anisotropies de densité totale et de densité du cerne 1965) mais seulement de 0,35 (non significative) entre 1 et 3 (anisotropies de densité totale et de largeur du cerne 1965) et entre 2 et 3 (anisotropies de densité et de largeur du cerne 1965). La valeur faible de cette dernière corrélation semblerait prouver que la dissymétrie en densité et celle en croissance radiale procèdent de phénomènes internes relativement différents (1).

\subsection{Corrélations entre moyennes de familles}

A la différence de ce que nous avons pu constater au niveau du seul cerne 1965 pour la corrélation génétique, la corrélation des moyennes des 9 familles pour l'anisotropie absolue Est-Ouest des largeurs des cernes et de la densité (en moyenne 4 cernes) est assez élevée, soit 0,82 (probabilité inférieure à 0,01 ). Cela provient en partie du fait qu'il s'agit de l'anisotropie absolue dans ce cas au lieu d'anisotropie relative dans le cas des cernes, mais, en fait, la corrélation des moyennes de familles pour l'anisotropie relative Est-Ouest est elle aussi assez élevée $(r=0,71)$. Il faut donc être très prudent quand on estime des paramètres génétiques sur les valeurs d'une seule année, où les conditions climatiques ont pu être très particulières.

Une autre corrélation de moyennes de familles intéressante à noter est celle de l'anisotropie absolue Est-Ouest de la densité avec la valeur moyenne des densités Est et Ouest $(r=0,72$ soit $0,01<p<0,05)$ et avec la densité Est $(r=0,79$ soit $p<0,01)$, alors que la liaison n'est pas significative avec la densité Ouest. Ceci reflète sans doute le fait que la dispersion des valeurs est plus forte à l'Est qu'à l'Ouest (variance de 126 pour la densité Est contre 99 pour la densité Ouest), et qu'en même temps les valeurs Est et Ouest sont fortement corrélées. Par conséquent, la sélection pour une forte densité estimée, soit d'après la moyenne des densités Est-Ouest, soit d'après la densité Est, aurait pour effet d'augmenter l'anisotropie Est-Ouest.

\subsection{Corrélations entre moyennes de provenances}

Dans l'essai D 440, on disposait de familles de demi-frères n'ayant aucun lien de parenté entre elles et établies dans un dispositif statistique avec répétitions et répartition au hasard. Dans le cas des Arrouilles, les provenances sont composées, ou bien d'une famille de demi-frères maternels, ou bien de plusieurs familles de demifrères maternels pouvant être parentes par les pères. Cette imprécision ne nous permet pas de définir nettement ce que représentent les variances et par conséquence les corrélations. Nous donnerons toutefois deux des corrélations calculées dans ce cas ; la corrélation, pour les moyennes de provenances, entre les valeurs de l'anisotropie de densité (mesurée par les différences entre les densités moyennes Est et Ouest) et de l'anisotropie de largeur de cernes (mesurée également par les différences entre

(1) Sur 237 plants, il y a 42 plants où le rapport des densités du cerne 1965 Est/Ouest est inférieur à 1 alors que le rapport des largeurs est supérieur a 1 , et 25 plants ou la situation est inverse: done plus du quart des arbres présentent des anisotropies opposées pour la largeur des cernes et la densité. On note en outre que la largeur de cernes est plus souvent supérieur à l'Est que ne l'est la densité. 
les largeurs de cernes) n'est pas significativement différente de zéro ( $r=0,135$ avec $r_{0,05}=0,404$ ), contrairement à celle calculée plus haut pour les moyennes de familles. Ceci est d'autant plus étonnant que l'on devrait au moins voir une liaison en ne conservant que les provenances landaises, ce qui n'est pas le cas.

Par contre, si l'on étudie la corrélation entre l'anisotropie de densité et la seule densité Est, on a, comme dans le cas des familles landaises, une corrélation significative ( $r=0,467$, soit $0,01<p<0,05$ ), la liaison ne se manifestant pas avec la densité Ouest.

\section{CONCLUSIONS}

L'anisotropie est donc un phénomène très général chez le Pin maritime des Landes ; le plus souvent, elle atteint son amplitude maximale dans le sens Ouest-Est, qui correspond aussi à celui du grand axe du méplat des arbres, cependant que l'inclinaison la plus courante des tiges est également dirigée vers l'Est ; mais des exceptions assez nombreuses existent, et les trois phénomènes peuvent se produire suivant trois orientements différents.

Si l'on fait le bilan des caractéristiques du bois de la face Est, on constate qu il présente beaucoup d'analogies avec le bois de compression, tel qu'il est décrit dans la littérature : plus grande largeur des cernes, densité plus élevée, fibres plus courtes, retrait longitudinal plus important, et parfois, mais pas toujours, présence de méats intercellulaires et insertion en spirales très aplaties des microfibriles de la couche moyenne de la membrane secondaire. Il s'y ajoute des critères originaux mais qui ne sauraient surprendre : rétractibilité transversale plus faible (à rapprocher de l'inclinaison plus forte des micelles) et rendement en fibres réduit (dû sans doute à une teneur en lignine plus élevée).

Cependant, le retrait longitudinal, quoique supérieur à celui de l’Ouest, est très loin d'atteindre les pourcentages que I'on trouve habituellement dans le vrai bois de compression, et de plus l'anisotropie des largeurs de cernes est assez peu liée à celle des densités.

Ceci amène à penser que la littérature englobe sous le vocable « bois de compression ” divers bois anormaux d'origines différentes dont les caractéristiques peuvent parfois coinncider, mais qui sont susceptibles de se différencier suivant d'autres critères. Dans le cas présent en particulier, l'anisotropie des largeurs de cernes, qui est à l'origine de l'excentricité de la moelle, et celle des densités pourraient ne pas avoir exactement la même origine physiologique : la première serait due à l'action des vents dominants qui soufflent de l'Ouest (I'hypothèse reste à vérifier mais concorde bien avec les résultats expérimentaux de LARSON) ; la seconde dépendrait davantage de l'inclinaison de la tige qui, en dehors des cas oủ elle est provoquée par l'héliotropisme, résulte de forts coups de vent inclinant l'arbre dans sa totalité, de façon brutale et irréversible, dans une direction qui est aussi, assez souvent mais pas toujours, opposée à l'Ouest. De toute façon, le vent a une action prépondérante.

11 reste à expliquer pourquoi le bois de l'Est n'a pas un retrait longitudinal aussi élevé que le bois de compression typique ; ce peut être dû, au moins en partie, au fait que le Pin maritime des Landes a une croissance, en hauteur, et surtout en circonfé- 
rence, qui dure plus longtemps que celle des autres essences résineuses ; l'afflux d'auxine, qui a été reconnue responsable de la formation du bois de réaction, serait certes plus important sur le côté Est vers lequel les cimes sont déportées et qui est donc susceptible de renfermer de ce fait une plus grande quantité de bourgeons générateurs d'hormones de croissance ; mais cet afflux irait en diminuant tout au long de la période de végétation, en sorte qu'une forte partie des accroissements annuels du côté Est serait formée de bois à orientation micellaire presque normale, conduisant à un retrait longitudinal moyen qui, tout en étant plus élevé qu'à l'Ouest, ne serait pas excessif.

Au point de vue technologique, le bois de la face Est ne présente pas d'inconvénients majeurs pour des utilisations traditionnelles telles que le sciage : son retrait axial, qui n'est pas assez important pour occasionner un déclassement des produits, est compensé par une rétractibilité dans le sens transversal inférieure à la normale, et par une dureté plus élevée, avantageuse pour certaines catégories d'emplois (en parqueterie par exemple) ; de plus, bénéficiant d'accroissements annuels plus larges, il renferme un volume plus important de bois sans nouds.

Pour des usages papetiers au contraire, il est certain qu'à l'unité de poids de matière sèche, le bois de l'Est est notablement inférieur à celui de l'Ouest, ayant à la fois des fibres plus courtes et un rendement en fibres plus faible ; la différence est telle que si les côtés Est et Ouest des mêmes arbres faisaient l'objet de cuissons séparées, la qualité des papiers obtenus serait assez médiocre pour les premiers.

Sur le plan pratique, on voit difficilement comment remédier à l'anisotropie du Pin maritime par des opérations purement culturales ; on pourrait songer à des coupes de faible étendue de façon que les arbres bénéficient de l'abri latéral des peuplement voisins, mais l'intérêt économique d'une telle sylviculture serait très discutable. La seule voie possible semble être celle de l'amélioration génétique par sélection d'arbres moins anisotropes, au moins en ce qui concerne la largeur des cernes. L'étude des corrélations génétiques a d'ailleurs montré que la sélection pour de fortes densités peut conduire à une aggravation de l'anisotropie si l'on sélectionne d'après la densité de l'Est, et, en définitive, il sera toujours préférable de sélectionner les arbres d'après la densité la plus basse.

En tout état de cause, l'héritabilité significative de l'anisotropie absolue doit être prise en considération, et si l'on considère que l'homogénéité d'un bois est un critère de qualité, on peut avoir avantage à rechercher des individus ou des provenances dotées d'une faible anisotropie, d'autant qu'elle présente une corrélation génétique positive avec la forme et la hauteur des arbres.

Le méplat des arbres, c'est-à-dire leur forme elliptique avec le grand axe dirigé aussi dans le sens Ouest-Est, présente aussi un intérêt en dendrométrie : quand on mesure des diamètres et non des circonférences, il faut savoir que le diamètre EstOuest est supérieur de $5 \%$ au diamètre Nord-Sud et que, par conséquent, on ne doit pas mesurer les diamètres suivant une orientation systématique.

De nombreuses recherches sont nécessaires pour mieux connaître les phénomènes conduisant à l'anisotropie : certaines sont en cours, comme celles visant à déterminer, à l'aide de microdendromètres, les rythmes de croissance saisonniers sur les côtés Est et Ouest d'une même tige, ou à étudier sur les greffes d'un même clône les différences de densité et de largeurs de cernes. D'autres sont à entreprendre : il serait 
en particulier nécessaire de mieux définir le bois normal, et de vérifier, par des expêriences de tuteurage, qu'il est en général représenté, à la largeur de cernes près, par le bois de l'Ouest. Ces recherches devraient d'ailleurs permettre d'approfondir nos connaissances dans le domaine de la forme des arbres, aussi bien tige que houppier, qui est un facteur très important de qualité.

\author{
Resu pour publication en aoüt 1967
}

\title{
SUMMARY
}

\section{Studies on ANisotropy of Pimus pinaster IN THE LANDES fOREST}

PINus pinaster in the Landes Forest generally has wider annual rings and wood of higher specific gravity on the eastern than on the western side of the bolc. This anisotropy is observed as well near the Ocean as very far from the shore, as well at the ground level as at anyone level of the stem, and as well on 4 years old seedlings as on older trees; the phenomenon is present in all provenances, but perhaps it is less severe in the mediterranean provenances than in the Landes ones.

On an average, the anisotropy of ring width is heavier than that of specific gravity, and furthermore there is no significative correlation between them.

The Pines exposed to the wind blowing from the Ocean have significantly higher heterogeneity than those which are protected by an adjacent crop ; elsewhere, there is a significant correlation between ring width anisotropy and the angle of inclination of the bole on the vertical.

The wood of eastern side of Pinus pinaster in the Landes has most of the features of compression wood, with the exception of high longitudinal shrinkage ; thus, it has no technological defect, serious enough to forbid utilizations like saw timber, but its low pulp yield and low mean fibrelength give rather poor characteristics for pulp and paper industries.

The heritability of absolute ring width anisotropy, i.e. of the ratio of the larger to the smaller of the radius East and West is significant. Elsewhere, a significant correlation has been found, within a progeny test, between the values of the absolute specific gravity anisotropy and either the eastern, or the average of eastern and western specific gravity. It probably would be possible to reduce anisotropy by genetic means, but an improvement programme based on a research for high eastern specific gravity could lead to an aggravation of the specific gravity anisotropy.

\section{ZUSAMMENFASSUNG}

\section{UNTERSUCHUNGEN ÜBER diE ANISOTropie von Pinus pinaster IM WALdGFBIET der LANDES}

Die Seestrandkiefern Pimus pinaster Ait, in den « Landes $»$ haben im allgemeinen auf der Ostseite der Stämme breitere Jahrringe und eine höhere Rohdichte des Holzes als auf der Westseite. Man finde diese Anisotropie sowohl an Bäumen die in Küstennähe, als auch bis zu hundert kilometer im Landesinneren wachsen : desweiteren beobachtet man diese Anisotropie nicht nur in Stock- oder Brusthöhe, sondern durchgehend bis ins Kroneninnere ; man findet sie bereits an vierjährigen Pflanzen und gleichfalls an allen herkünften, wobei jedoch die mediterranen Provenienzen eine vielleicht etwas geringere Häufigkeit als jene der \& Landes $"$ aufzuweisen scheinen.

Im allgemeinen ist die Anisotropie der Jahrringbreiten ausgeprägter als jene der Rohdichteว und es besteht ausserdem keine signifikative Beziehung zwischen diesen beiden Anisotropien.

Die den vorherrschenden Seewinden ausgesetzten Seestrandkiefern weisen eine stärkere Anisotropie der Rohdichte auf als jene, die im Windschutz dieser Westwinde wachsen ; diese Unterschiede sind statistisch signifikativ. Desgleichen besteht eine signifikative Beziehung zwischen der Anisotropie der Jahrringbreiten und dem Winkel der Stammabweichung von der Senkrechten. 
Alles in allem kann man sagen, dass das Holz an der Ostseite der Stämme die wesentlichen Merkmale des Druckholzes aufweist, jedoch mit Ausnahme eines exzessiven Schwundes in der Längsrichtung. Man kann daher sagen, dass das Holz keine verborgenen technologischen Fehler für die meisten Nutzholzverwertungen besitzt, jedoch ist seine Verwendung in der Papierindustrie wegen der geringen Anzahl-und Mittellänge der Fasern nicht sehr vorteilhaft.

Von den verschiedenen berechneten Heritabilitäten war nur die absolute Anisotropie der Jahrringbreiten Ost - West, d.h. das Verhältnis des grössten zum kleinsten Radius Ost - West statistisch signifikativ. In einer Nachkommenschaftsprüfung, die eine gewisse Anzahl Familien von Halbbrüdern (= demi-fratrie) umfasste, konnte für die Familienmittelwerte eine signifikante Korrelation zwischen der absoluten Anisotropie Ost - West der Rohdichte und der Rohdichte Ost oder dem Mittelwert der Rohdichten Ost und West beobachtet werden. Es müsste daher mōglich sein eine züchterische Verminderung der Anisotropie zu erreichen, man muss jedoch andererseits beachten, dass eine Auslese die sich auf eine hohe Rohdichte Ost oder eine hohe mittlere Rohdichte begründet alle Voraussetzungen besitzt, die Rohdichteanisotropie zu erhöhen.

\section{RÉFÉRENCES BIBLIOGRAPHIQUES}

Buscien et Munci, 1929. The structure and life of Forest trees, $3^{\circ}$ éd. Chapman et Hall, Londres. Dadswell H.E., Wardrop A.B., 1949. What is reaction wood ? Austral. Forest., 13, 22-23.

Ewart A.J., Mason-Jones A.J., 1906. The formation of redwood in conifers. Amn. Bot. (London). 20, 201-204.

Iu. G., 1966, Recherches sur l'amélioration génétique du Pin maritime. Ann. Sci. forest., 23, 4 , $769-946$.

JaCCARD P., 1919. Nouvelles recherches sur l'accroissement en épaisseur des arbres, 200 pp. Mém. Fondation Schmyder won Wartensee, Zurich.

Jaccard P., 1938. Exzentisches Dickenwachstum und anatomisch - histologische Differenzierung des Holzes. Ber. Schweiz, Bot. Gesell., 48, 491-537.

LARsoN P.R., 1965. Stem form of young Larix as influenced by wind and pruning. Forest. Sci., 2 (4) $412-24$

Parrot L., 1960. De la variabilité génétique de la densité du bois chez l'Epicéa (Picea excelsa Link). Ann. Ec, nation, Eaux et Forêts, 17 (3), 267-375.

Pot.ge H., 1966. Etablissement des courbes de variation de la densité du bois par exploration densitométrique de radiographies d'échantillons prélevés à la tarière sur des arbres vivants. Ann. Sci. forest., 23, 1 - 206.

Polge H., Itıy G., 1967. Héritabilité de la densité et corrélations avec la croissance étudiées à l'aide de tests non destructifs sur plants de Pin maritime de quatre ans. Silv. genet. (sous presse).

Sinnot E.W., 1952. Reaction wood and the regulation of tree form. Amer. $J_{.}$, Bot, , 39, 69-78.

Strasburger, 1891. Cité par Priestley J.H. et Tong D. (1927). Proc. (Sci. Sec.) Leeds Phil. Soc., 1 (5), 190 .

WARDROP A.B., 195I. Cell wall organisation and the properties of the xylem-I. Cell wall organisation and the variation of breaking lead in tension of the xylem in conifer stems. Austral. J. Sci. Res., Ser. B., 4, 391-414.

Wareing PF., HanNey, C.E.A., and DigBy J., 1964. The role of endogenous hormones in cambial activity and xylem differentiation. in The formation of wood in forest trees, 323-344. ZIMMERMANN M.H., Academic. Press., New York.

Wershing H.F., Bailey J.W. Seedling as experimental material in the study of redwood in Conifers. J. Forest., 40, 411-414. 